\title{
Mesembryanthemum crystallinum L. als dermatologisch wirksame Heilpflanze - erste Ergebnisse aus 3 Pilotstudien
}

\author{
Christa Raak $^{a, b} \quad$ Friedrich Molsberger $^{b} \quad$ Ulrike Heinrich $^{c} \quad$ Mathias Bertram $^{d}$ \\ Thomas Ostermann ${ }^{a}$ \\ a Institut für Integrative Medizin, Universität Witten/Herdecke, Herdecke, Deutschland; \\ ${ }^{b}$ Hochschule für Gesundheit und Sport, Technik und Kunst, Berlin, Deutschland; \\ ${ }^{\mathrm{c}}$ Institut für experimentelle Dermatologie, Universität Witten/Herdecke, Herdecke, Deutschland; \\ ${ }^{d}$ Lehrstuhl für familienorientierte und gemeindenahe Pflege, Universität Witten/Herdecke, Herdecke, Deutschland
}

\section{Schlüsselwörter}

Phytotherapie - Hautkrankheiten .

Mesembryanthemum crystallinum L. · Pilotstudie

\section{Zusammenfassung}

Hintergrund: Neben vielen bekannten Heilpflanzen der rationalen Phytotherapie gibt es eine Vielzahl von Pflanzen, deren Wirkung nur unzureichend erforscht oder deren Wirkpotential in Vergessenheit geraten ist. Zu diesen Pflanzen gehört die Kristall-Mittagsblume (Mesembryanthemum crystallinum L.). In der vorliegenden Arbeit werden 3 Pilotstudien vorgestellt, die die dermatologische Wirkung des Mittagsblumenextrakts (Mesem-Creme) näher untersuchen. Material und Methoden: In 2 Anwendungsbeobachtungen wurde zunächst das Wirkprofil der Mesem-Creme näher untersucht. Neben einer retrospektiven Anwenderbefragung wurde außerdem bei 6 Rollstuhlfahrern eine prospektive Befragung zur Veränderung des Hautstatus im Prä-post-Design durchgeführt. In einer dritten placebokontrollierten Pilotstudie wurde die Hautfeuchtigkeitsveränderung mittels Corneometermessung an 6 Probanden mit trockener Haut analysiert. Ergebnisse: Im Vergleich zur unbehandelten Haut konnte in der Corneometer-Studie eine signifikante Steigerung der Hautfeuchtigkeit mit der Mesem-Creme erzielt werden $(25,8 \pm 5,8$ vorher zu 46,6 $\pm 9,2$ nachher vs. $26,3 \pm 6,0$ vorher zu $33,8 \pm 6,0$ nachher) sowie ein statistischer Trend ( $p=0,11$; $t$-Test) im Vergleich zur Basiscreme ohne Mesem-Zusatz (25,1 $\pm 4,7$ vorher zu 41,9 \pm 7,3 nachher). Dieser Effekt der Steigerung der Hautfeuchtigkeit wurde auch in den Anwendungsbeobachtungen verifiziert. Die Hauttrockenheit hatte sich bei 17 von 29 Probanden "sehr verbessert" oder "verbessert". Auch die Anwendungsbeobachtung bei einer Kohorte von 6 Rollstuhlfahrern ergab signifikante Verbesserungen des Hautzustands in Bezug auf die Weichheit und Trockenheit der Haut im Vorher-nachher-Vergleich. Schlussfolgerung: In dieser Arbeit wurde die Mesem-Creme durch 2 Anwendungsbeobachtungen im Hinblick auf Wirkung, Produkteigenschaften und Verträglichkeit und durch den etablierten Corneometertest auch in Hinblick auf objektivierbare Hautfeuchtigkeitsveränderungen evaluiert. Aufgrund der geringen Fallzahlen gibt es trotz der ermutigenden Ergebnisse Limitationen in der Ergebnisinterpretation. Zudem sollte das Design künftiger Studien klarer sein und den hautfeuchtigkeitsverbessernden Effekt der Mesem-Creme besser fokussieren.

\section{Keywords}

Phytotherapy - Skin disease .

Mesembryanthemum crystallinum L. . Pilot study

\section{Summary}

Mesembryanthemum crystallinum L. As Dermatologically Effective Medicinal Plant - First Results from 3 Pilot Studies

Background: Apart from well-known medical plants of rational phytotherapy there is a variety of plants, such as Mesembryanthemum crystallinum L., whose potential effects are not examined sufficiently to date. Therefore, in 3 pilot studies we investigated the dermatological effect of an extract of $M$. crystallinum (Mesem Cream). Materials and Methods: In a retrospective user survey and a prepost study with 6 persons in a wheelchair the efficacy profile of Mesem Cream was investigated. In a third placebo-controlled pilot study changes in skin hydration was measured using corneometer measurement in 6 healthy volunteers with dry skin. Results: Compared to untreated skin, corneometer measurement revealed a significant increase of skin hydration with Mesem Cream (25.8 \pm 5.8 pre to $46.6 \pm 9.2$ post treatment vs. $26.3 \pm 6.0$ pre to $33.8 \pm 6.0$ post treatment) as well as a statistical trend $(\mathrm{p}=0.11$; $\mathrm{t}$-Test) compared to basic cream $(25.1 \pm 4.7$ pre to $41.9 \pm 7.3$ post treatment). Improvement in skin hydration was also found in 17 of 29 survey participants. Further, the volunteers in the wheelchair showed significant improvements in their subjectively rated skin softness and hydration level. Conclusion: These pilot studies investigated skin hydrating effects of the traditional medical plant $M$. crystallinum. Although the results were promising, they have to be interpreted with caution in particular due to low sample size. Further, study design should be more clear-cut and focus skin hydration as a main outcome parameter in more detail.

\section{KARGER}

Fax +497614520714 (c) 2014 S. Karger AG, Freiburg

$1661-4119 / 14 / 0216-0366 \$ 39.50 / 0$
Christa Raak, BSc

Institut für Integrative Medizin

Universität Witten/Herdecke

Gerhard-Kienle-Weg 4, 58313 Herdecke, Deutschland

christa.raak@uni-wh.de 


\section{Einleitung}

Neben vielen bekannten Heilpflanzen der rationalen Phytotherapie wie Johanniskraut oder Arnica gibt es eine Vielzahl von Pflanzen, deren Wirkung nur unzureichend erforscht ist oder deren Wirkpotential in Vergessenheit geraten ist. $\mathrm{Zu}$ diesen Pflanzen gehört die Kristall-Mittagsblume (Mesembryanthemum crystallinum L.). Der Name Mesembryanthemum leitet sich von Mesembria (gr.) = Mittag und anthemon (gr.) = Blüte oder Blume ab. Zunächst wurde davon ausgegangen, dass sich die Blüten nur mittags öffneten, später wurden jedoch auch nachtblühende Sorten entdeckt. Aktuell bezieht sich der Name auf «Blüte mit einer Frucht in der Mitte» $($ mesos $=$ Mitte, bryon $=$ Frucht $)$. Der Name crystallinum (cryophytum $=$ Eis gr.) beschreibt die zahlreichen epidermalen Blasenzellen auf den Stängeln und Blättern. Dadurch scheint die Pflanze wie vereist (Abb. 1).

Die Mittagsblume gehört zu den Halophyten und ist im deutschsprachigen Raum auch als Eisblume, Eiskraut, Kristall-Mittagsblume, Kristallkraut oder Sodapflanze bekannt [1]. Der Name Sodapflanze geht darauf zurück, dass die Asche der Mittagsblume bis zu 40\% kohlensaures Natron enthält und daher auf den Kanarischen Inseln im 19. Jahrhundert zur Gewinnung von Soda (Natriumcarbonat) genutzt wurde [2]. Das trockene Kraut liefert nach Bergs Handbuch der pharmazeutischen Botanik 42\% Asche, die aus 34\% in Wasser löslichen Natronsalzen mit wenig Kalisalzen und $8 \%$ erdigen Salzen besteht (Soda alicantina). Auch Madaus berichtet von einem auffallend hohen Aschegehalt (30-50\% der Trockensubstanz, davon die Hälfte $\mathrm{K}_{2} \mathrm{O}$ ) [3].

Nach Madaus [3] enthalten die Blätter der M. crystallinum Zitronensäure, Apfelsäure, Oxalsäure und Phosphorsäure. In der Übersichtsarbeit von Wende et al. [4] werden für M. crystallinum weitere, in Tabelle 1 zusammengefasste Inhaltstoffe angegeben. Die in der Pflanze enthaltenen Flavonoide und Betacyane schützen diese vor extremer Sonnenbestrahlung; hydrophile Substanzen wie Zuckeralkohole, Fruchtsäuren, Mineralstoffe, Aminosäuren wirken osmotisch aktiv und minimieren den Wasserverlust der Pflanze in Dürreperioden [4]. Ibtissem et al. [5] identifizierten in M. crystallinum das natürliche Antioxidans Butylhydroxytoluene (BHT), das in der
Pflanze unter Stress wie z.B. Salinität angehäuft wird. Unter photosynthetischem Stress findet eine Anhäufung von Flavonoiden in der Pflanze statt.

Als Halophyt ist es der Mittagsblume außerdem gelungen, sich an sehr hohe Salzkonzentrationen zu adaptieren. Sie scheidet aufgenommene Salze über ihre Blätter in die Zellen auf den Blattoberflächen aus, oder aber sie lagert die Salzionen in die Vakuolen der Zellen ein. Ist die M. crystallinum durch hohe Salzkonzentrationen, Dürreperioden oder Hitze gestresst, so schaltet sie auf den sogenannten CrassulaceenSäurestoffwechsel (kurz CAM-Stoffwechsel) um. Dafür verkleinert die Pflanze die Öffnung ihrer Stomata tagsüber, und nur nachts wird $\mathrm{CO}_{2}$ fixiert. Das aufgenommene $\mathrm{CO}_{2}$ wird dann vorübergehend an Phosphoenolpyruvat (PEP) gebunden und erst bei der nächsten Lichteinstrahlung weitersynthetisiert [6]. Dieser Mechanismus erfolgt über die epidermalen Blasenzellen (sogenannte Idioblasten) (Abb. 2): bei Wassermangel kommen sie als Wasserspeicher spontan zum Einsatz. Der Turgor der Blase wird verringert und damit auch die Abstände zwischen den Blasen, sodass die Fläche schrumpft, die mit den Stomata zum Gasaustausch zur Verfügung steht [7]. Dieser Mechanismus steigert die Wassernutzungseffizienz der $M$. crystallinum (und anderer CAM-Arten) bis um das Fünffache gegenüber anderen, nicht CAM-Arten [8].

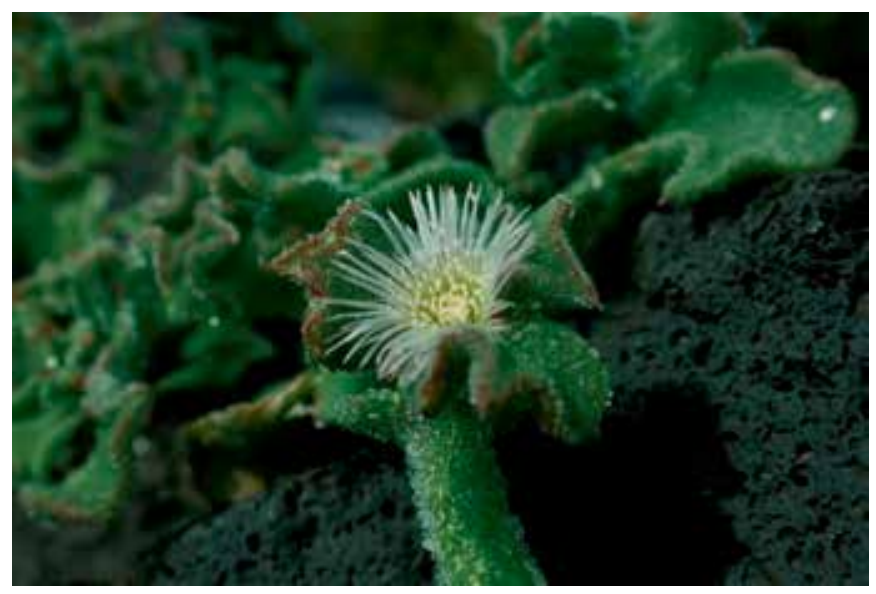

Abb. 1. Blüte der Mittagsblume (Copyright C. Raak, 2013).
Tab 1. Inhaltstoffe von Mesembryanthemum crystallinum L.; aus [4]

\begin{tabular}{ll}
\hline Substanzklasse & Primär- und Sekundärstoffe \\
\hline Fruchtsäuren & $\begin{array}{l}\text { Zitronensäure, Fumarsäure, Äpfelsäure, Oxalsäure, Bernsteinsäure, } \\
\text { Weinsäure, Ascorbinsäure }\end{array}$ \\
Flavonoide & Mesembryanthin \\
Betacyane & Betanin, Betanin-Feruloylderivate, Betanidin-5-O-di- und -triglucoside \\
Aminosäuren & L-Glycin, L-Leucin, L-Isoleucin, L-Phenylalanin, L-Serin, L-Tyrosin, \\
& L-Arginin, L-Threonin, Hydroxy-L-Prolin, L-Prolin \\
Mono- und & Glucose, Fructose, Galactose, Glucuronsäure, Rhamnose, Saccharose \\
Disaccharide & \\
Polysaccharide & methylierte Rhamnogalacturonane \\
Zuckeralkohole & Myo-Inositol, Ononitol, Pinitol \\
\hline
\end{tabular}




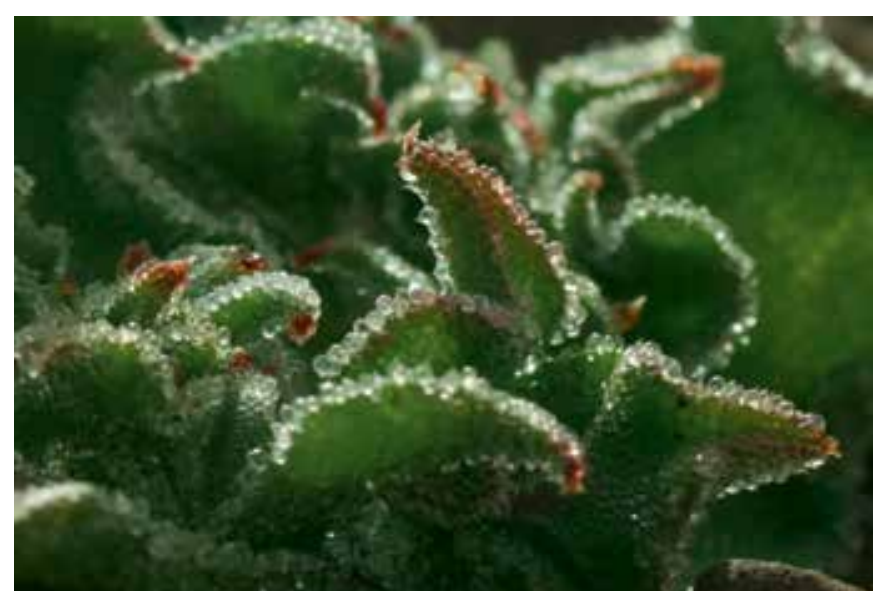

Abb. 2. Epidermale Blasenzellen der Mittagsblume (Copyright C. Raak, 2013).

Die traditionelle medizinische Verwendung bezieht sich vor allem auf Erkrankungen der harnbildenden und -ableitenden Organe. So wurde M. crystallinum von Madaus unter Berufung auf Lieb (1785) als spezifisches Mittel bei Anhäufung von Schleim im Unterleib und Blasenproblematiken angegeben, aber auch bei Gallenleiden und Keuchhusten empfohlen [9]. Hahnemann erwähnte die Mittagsblume in seinem Apothekerlexikon von 1798 [10]: «Des Krautes (Mesembryanth. cristall.) frisch ausgepresster Saft, welcher wässerich ekelhaft schmeckt, hat man, mit wenig Gewährleistungen unterstützt, gegen Wassersucht und Harnstränge, als ein harntreibendes, verdünnendes Mittel gerühmt.» 1801 veröffentlichte Hufelands Mitarbeiter Wendt eine ausführliche Arbeit über $M$. crystallinum und bestätigte die Wirkung bei urologischen Erkrankungen, Pertussis und Tuberkulose [3].

Von Lincke [11] wurde 1841 in Bezug auf die Wirkung der M. crystallinum folgendes geschrieben: «Das Kraut, welches man anwendet, soll kühlende, auflösende und harntreibende Wirkungen besitzen. Man hat den frisch ausgepressten Saft bei Abdominalinfarcten, Anhäufung von Galle, Keuchhusten und besonders bei Dysurie benutzt.» Georg Friedrich Most beschreibt 1843 in seiner Enzyklopädie der Volksmedicin [12] die kühlende Wirkung folgendermaßen: «Einige gequetschte Eiskraut-Blätter, mit einer kleinen Binde auf die Pulsgegend bei Fieberkranken gegen die Fieberhitze aufgebunden, ist ein beliebtes, nicht unwirksames Volksmittel».

Über die Erfahrungsberichte von Marschke [13] fanden Extrakte der M. crystallinum in der jüngeren Vergangenheit als Hautpflegecreme für trockene Haut Verwendung. «Marschke begann auf der Finca des Anthroposophischen Zentrums von Lanzarote mit dem glitzernden Kraut zu experimentieren: Sie sammelte die sukkulenten, fleischigen Blätter dieser Pflanze, die ihren Stoffwechsel perfekt an die intensive Sonneneinstrahlung, die Trockenheit und den Salzstress angepasst hat, presste den frischen Pflanzensaft aus und wendete ihn unverdünnt, oder als Auszug in Bädern, bei den verschie- densten Hautkrankheiten an. Außerdem entwickelte sie zusammen mit Apothekern eine sehr effektive Salbe mit Auszügen aus der Kristall-Mittagsblume.» [2]

In ihrer Studie von 2008 [14] bestätigten Bouftira et al. den Gebrauch des Mittagsblumenextrakts für eine kosmetische Rezeptur mit Inhaltsstoffen wie Superoxiddismutase (SOD), einem kupferhaltigen Enzym. Im menschlichen Organismus kommt es in den Erythrozyten vor und dient der Entfernung von Sauerstoffradikalen. In Kosmetika wird SOD in Cremes eingesetzt, die dem Hautschutz dienen und der Hautalterung entgegenwirken sollen [4]. Auch Greco berichtet über eine Creme auf Basis der Kristall-Mittagsblume zur medizinischen Hautpflege [15].

In der hier vorliegenden Arbeit werden 3 Pilotstudien vorgestellt, die die dermatologische Wirkung eines Mittagsblumenextrakts näher untersuchen.

\section{Material und Methoden}

Die hier beschriebenen Studien untersuchen eine Hautcreme, die am Gemeinschaftskrankenhaus Herdecke hergestellt wird. Sie basiert auf dem filtrierten Presssaft der frisch geernteten Pflanze, der mit 25\% Alkohol versetzt haltbar gemacht und mit einer Basiscreme bestehend aus Aqua, Prunus dulcis, Glycerin zur Mesem-Creme verarbeitet wird. Die Mesem-Creme enthält eine Endkonzentration von 3,4\% Alkohol.

\section{Studie 1: Retrospektive Anwendungsbeobachtung}

Bei der ersten Studie handelte es sich um eine retrospektive Befragung von Personen, die die Mesem-Creme nutzen, weil sie subjektiv dadurch eine Verbesserung ihres Hautstatus erleben. Hierzu zählen Menschen mit trockener bis zu stark schuppender, psoriatischer Haut, leichter altersbedingter Akne bis zu juckenden und brennenden Dermatosen sowie Personen mit atopischen Hautekzemen (z.B. Neurodermitis). Die Studienteilnehmer umfassten Patienten des Gemeinschaftskrankenhauses Herdecke, die aufgrund von Hautproblemen Mesem-Creme durch Pflegepersonal oder Ärzte erhalten haben, sowie Personen, an die Mesem-Creme durch die Krankenhausapotheke bzw. die «Sonnenschein Apotheke Herdecke» per Post verschickt wurde. Insgesamt wurde ein Kollektiv von 82 Benutzern befragt.

\section{Studie 2: Prä-post-Anwendungsbeobachtung}

Neben der retrospektiven Befragung wurde in einer zweiten Anwendungsbeobachtung bei 6 Rollstuhlfahrerinnen (Erstnutzerinnen im Alter zwischen 30 und 61 Jahren; Mittelwert (MW): $47 \pm 12$ ) aus der Abteilung für querschnittsgelähmte Patienten des Gemeinschaftskrankenhauses Herdecke eine prospektive Befragung im Prä-post-Design zur Veränderung des Hautstatus durchgeführt. Dabei wurden die in der ersten Anwendungsbeobachtung benutzten Fragebögen angewandt. Ziel war es, Veränderungsindikatoren zu identifizieren, die in nachfolgenden Studien Erfolg versprechend sein können.

\section{Studie 3: Corneometermessung}

Die dritte Studie war eine placebokontrollierte Pilotstudie, in der die Hautfeuchtigkeitsveränderung mittels Corneometermessung bestimmt wurde. Dabei handelt es sich um eine effiziente Methode, den Wassergehalt des Stratum corneums unter verschiedenen experimentellen Bedingungen zu bestimmen. Diese Methode weist eine hohe Reproduzierbarkeit auf und wird in zahlreichen Publikationen für Feuchtigkeitsnachweise als geeignet beschrieben [16]. Das hier verwendete Corneometer CM 825 (Courage + Khazaka electronic GmbH, Köln, Deutschland) misst die 
Hautfeuchtigkeit mittels einer kapazitiven Erfassungsmethode. Der Messkopf mit einem Durchmesser von $10 \mathrm{~mm}$ wird mit konstantem Auflagedruck auf die Messstelle aufgesetzt, wobei die Haut als Dielektrikum (d.h. als Isolationsschicht) des Kondensators wirkt. Je nach Wassergehalt in der Haut ändert sich dabei die Kapazität des Kondensators, sodass die Hautfeuchtigkeit durch die Kapazität bestimmt werden kann.

Die Probandinnen wandten die Mesem-Creme 2-mal täglich an einer Unterarm-Innenseite an. Auf der anderen Unterarm-Innenseite erfolgte die Anwendung der wirkstofffreien Grundlage (Basiscreme ohne Mesem-Zusatz). Darüber hinaus wurde ein weiteres Testfeld (Kontrolle) mit untersucht, an dem keinerlei Produktanwendung stattfand. Alle Probandinnen wurden angewiesen, im Verlauf der Prüfung ausschließlich die ihnen zur Verfügung gestellten Pflegeprodukte an den UnterarmInnenseiten zu verwenden und ihre sonst üblichen Hautreinigungsgewohnheiten beizubehalten. Die Messung der Hautfeuchtigkeit nach Ap plikation des jeweiligen Präparates (Mesem-Creme und Placebo) erfolgte mit dem Corneometer und im Vergleich zur unbehandelten Kontrolle vor Erstanwendung der Produkte sowie nach 4-wöchiger Anwendung.

\section{Eingesetzte Fragebogeninstrumente (Studien 1 und 2)}

Der Zustand der Haut wurde in einem Fragebogen mit den 7 Gegensatzpaaren «weich-hart», «trocken-feucht», «blass-gerötet», «schlaff-gespannt», «kalt-warm», «glatt-rau» sowie «angenehm-unangenehm » auf einer Skala von 1 bis 5 erfasst, wobei ein Wert von 3 die Mitte markierte.

Hautprobleme wurden mit den 8 Items «brennt», «juckt», «empfindlich», «fettig», «rissig», «schuppt», «schwitzt» und «klebt» erfasst. Ein Punktwert von 3 Punkten wurde für ein sehr starkes Zutreffen vergeben, 2 Punkte für ein starkes Zutreffen und 1 Punkt für ein mäßiges Zutreffen. Für die Antwortalternativen «gar nicht», «nicht relevant» und «Frage betrifft mich nicht» wurden 0 Punkte vergeben.

Die Eigenschaften der Mesem-Creme wurden mit den 4 Fragen «Wie lässt sich die Creme auf der Haut verteilen?», «Wie zieht die Creme ein?», «Wie ist die Verträglichkeit?», «Wie beurteilen Sie die Fettung auf der Haut?» erfasst. Als Antwortmöglichkeiten konnten die Kategorien «sehr gut», «gut», «ausreichend» und «schlecht» angekreuzt werden.

Als Parameter für die Verbesserung des Hautzustands wurden die Dimensionen «Trockenheit», «Juckreiz», «Kribbeln», «Brennen» sowie das «Hautgefühl nach Anwendung» verwendet. Als Antwortmöglichkeiten konnten die Kategorien «sehr verbessert», «verbessert», «unverändert» und «verschlechtert» angekreuzt werden.

\section{Ethische Aspekte}

Den Studien liegt ein positives Votum der Ethikkommission der Deutschen Gesellschaft für Pflegewissenschaft e.V. (Anwendungsbeobachtungen) bzw. der Ethikkommission der Universität Witten/Herdecke (Corneometer-Studie) zugrunde. Alle Teilnehmer wurden über Zweck und Ziel der Studien schriftlich aufgeklärt und willigten schriftlich ein, an der jeweiligen Studie teilzunehmen. Dem Datenschutz wurde durch Anonymisierung der Daten in vollem Umfang Rechnung getragen. Alle erhobenen Daten wurden für Dritte unzugänglich archiviert. Eine Verbindung zwischen erhobenen Daten und Informanten wurde damit ausgeschlossen.

\section{Fallzahlkalkulation}

Aufgrund des Pilotcharakters der Studien wurden die Untersuchungen zunächst an einem kleinen Probandenkollektiv von 6 Probanden orientierend durchgeführt. Unter Annahme einer Power von $80 \%$ und einem Signifikanzniveau von 5\% kann dadurch ein Unterschied in den Post-Werten der Corneometermessung von 12 Einheiten bei einer Standardabweichung von 7 Einheiten zwischen unbehandelter Kontrolle und Mesem-Creme signifikant nachgewiesen werden, was vor dem Hintergrund der bisherigen Erfahrungswerte mit der Mesem-Creme [15] als realistisch erschien. Da für den Vergleich mit dem Placebopräparat ohne Wirkstoff keine belastbaren Daten vorlagen, wurde im Rahmen einer Post-hoc-Analyse die notwendige Fallzahl bestimmt, unter der die gefun- denen Unterschiede zwischen Basiscreme und Mesem-Creme signifikant wurden. Für die Prä-post-Anwendungsbeobachtung der Veränderung der Hautfeuchtigkeit wurden aufgrund fehlender Erfahrungswerte und in Anlehnung an die Corneometer-Studie ebenfalls 6 Probanden angenommen und ebenfalls durch eine Post-hoc-Poweranalyse abgesichert («trocken-feucht»).

\section{Statistische Auswertungen}

Die Auswertung der Messergebnisse erfolgte zunächst mittels deskriptiver statistischer Verfahren. Unterschiede im Zeitverlauf wurden auf Itemebene durch Kontingenztafeln oder auf Skalenebene durch Mittelwertdifferenzen dargestellt. Durch inferenzstatistische Methoden (Chi-Quadrat-Test für nominale Daten bzw. Wilcoxon- oder t-Test für metrische Daten) wurden die hier gefundenen Unterschiede hinsichtlich ihrer Signifikanz bewertet. Auf weitere multivariate Analysen wurde aufgrund der geringen Fallzahl verzichtet.

\section{Ergebnisse}

\section{Retrospektive Befragung bei Nutzern der Mesem-Creme}

Insgesamt konnten Fragebögen von 29 Benutzern der Mesem-Creme ausgewertet werden, was einer Rücklaufquote von $35,4 \%$ entsprach. 21 Benutzer (75,9\%) gaben an, die Creme für sich selbst zu benutzen, während 7 Befragte $(24,1 \%)$ die Mesem-Creme für ihre Kinder bezogen hatten. Dementsprechend variierte die Altersverteilung von 1 bis 84 Jahren mit einem MW von 45,7 $\pm 26,5$ Jahren. 21 Anwender waren weiblich $(72,4 \%)$ und 8 männlich (27,6\%). 14 der Erwachsenen gaben Abitur als Schulabschluss an (66,7\%), 2 Befragte hatten einen Realschulabschluss (9,5\%) und 4 einen Hauptschulabschluss $(19,0 \%)$; 2 Befragte gaben keine Auskunft zu ihrer Schulbildung. Insgesamt waren 13 der 29 Befragten (44,8\%) entweder in Vollzeit $(\mathrm{n}=4 ; 13,8 \%)$ oder in Teilzeit berufstätig $(\mathrm{n}=9 ; 31,0 \%)$. Nicht berufstätig waren 14 Befragte $(48,3 \%$ inklusive der Kinder), 2 Befragte gaben keine Auskunft (6,8\%). Fast alle Befragten gaben an, täglich $(\mathrm{n}=27,93,1 \%)$ bzw. 3 - bis 4-mal wöchentlich $(\mathrm{n}=1 ; 3,4 \%)$ ihre Haut zu pflegen. Ein Befragter gab hierzu keine Auskunft. In Bezug auf die MesemCreme waren 5 Befragte Erstnutzer (17,2\%), 6 Befrage hatten die Creme bereits zuvor ab und zu benutzt (20,7\%) und 18 der Befragten (62,1\%) gaben an, die Mesem-Creme regelmäßig zu benutzen. Ein ähnlich großer Anteil von 22 Befragten (75,9\%) gab dabei an, weitere Hautpflegemittel zu benutzen.

Abbildung 3 zeigt die Angaben der Befragten zu Ihrem Hautzustand, wobei ein Wert von 3 einen ausgeglichenen Hautzustand beschreibt. Hier zeigt sich deutlich, dass die Befragten mit einem MW von 1,85 \pm 0,77 vor allem unter trockener Haut litten und damit signifikant von der Mitte abwichen ( $\mathrm{p}<0,001$, t-Test). Auch die Itempaare «schlaff-gespannt» und «kalt-warm» wiesen ebenfalls eine signifikante Abweichung von der Mitte auf («schlaff-gespannt»: 3,33 \pm 0,76 und «kalt-warm» 3,36 $\pm 0,86$ ), während alle anderen Items keine signifikante Abweichung von der Mitte zeigten.

In Bezug auf die Anwendung, Wirkung bzw. Wirksamkeit der Mesem-Creme auf die Haut ergeben sich die Ergebnisse aus Abbildung 4. Die Hauttrockenheit hatte sich bei 6 Pro- 


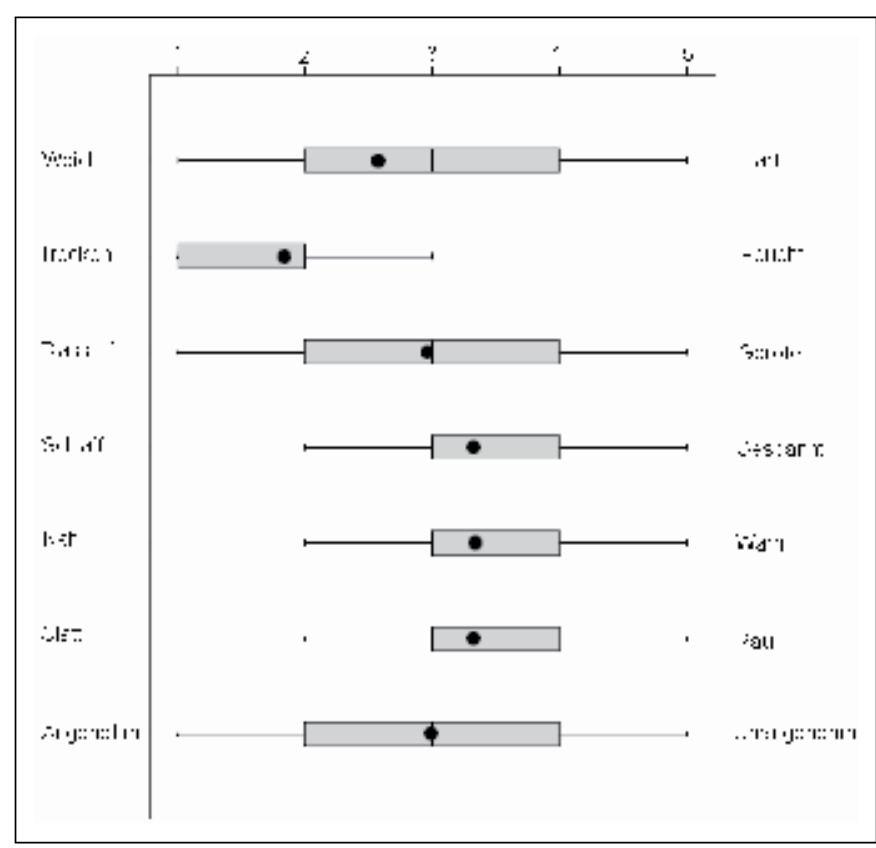

Abb. 3. Hautzustand der Befragten, erfasst auf einer Skala von 1 bis 5 . Ein Wert von 3 markiert die Mitte der Gegensatzpaare. Darstellung als Boxplots mit Mittelwertangabe (schwarzer Punkt).

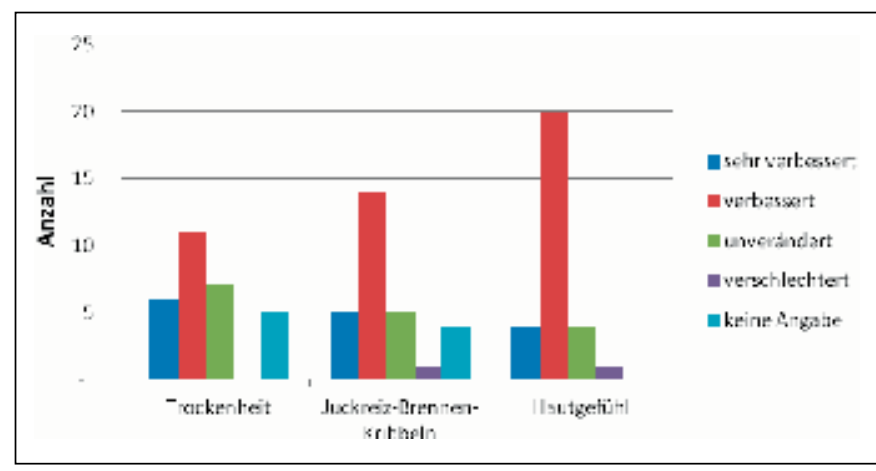

Abb. 4. Veränderungen des Hautzustands nach Anwendung der MesemCreme auf einer Likert-Skala von «sehr verbessert» bis «verschlechtert». Angabe in absoluten Häufigkeiten des Gesamtkollektivs ( $\mathrm{n}=29$ ).

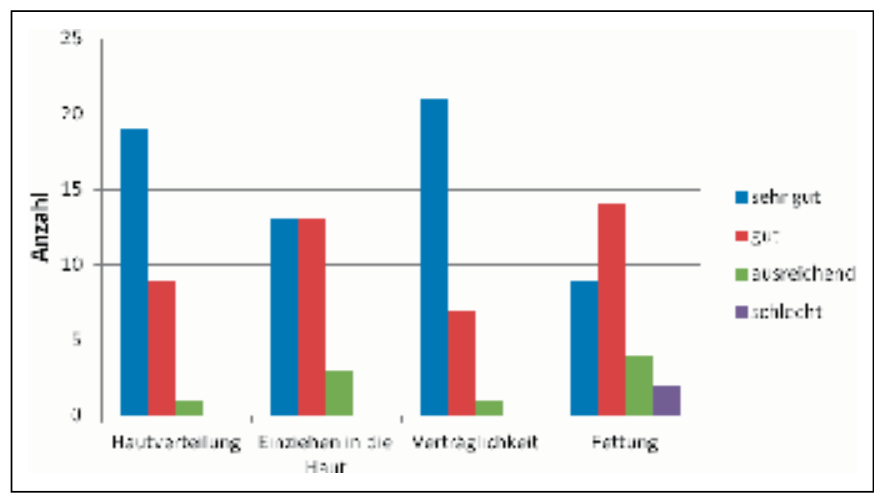

Abb. 5. Eigenschaften der Mesem-Creme auf einer Skala von $1=\ll \mathrm{sehr}$ gut» bis $4=$ «schlecht». Angabe in absoluten Häufigkeiten des Gesamtkollektivs $(n=29)$. banden sehr verbessert $(20,7 \%)$ und bei 11 Probanden $(37,9 \%)$ verbessert. Bei 7 Probanden gab es keine Änderung (24,1\%), und 5 Probanden (17,2\%) gaben keine Auskunft. Auch in Bezug auf Juckreiz, Kribbeln und Brennen gaben zwei Drittel der Probanden an, dass sich die Haut sehr verbessert $(\mathrm{n}=5 ; 17,2 \%)$ oder verbessert hatte $(\mathrm{n}=14 ; 48,3 \%)$. Bei 4 Probanden $(17,2 \%)$ gab es keine Veränderungen; ein Proband $(3,4 \%)$ gab eine Verschlechterung an, und 4 Probanden $(13,8 \%)$ gaben keine Auskunft. Das Hautgefühl nach Anwendung wurde von 4 Probanden $(13,8 \%)$ als sehr verbessert und von 20 Probanden $(69,0 \%)$ als verbessert angegeben. 4 Probanden $(13,8 \%)$ gaben ein unverändertes Hautgefühl an und ein Proband (3,4\%) eine Verschlechterung.

In Bezug auf die Eigenschaften der Creme wurden die in Abbildung 5 dargestellten Angaben gemacht. Insgesamt weist die Creme durchweg sehr gute Anwendungseigenschaften auf. Sowohl die Verteilung der Creme auf der Haut als auch die Verträglichkeit wurde von fast allen Teilnehmern als «sehr gut» oder «gut» empfunden (jeweils 28 von 29 Teilnehmern). Je 13 Probanden (44,8\%) berichteten, dass die Creme «sehr gut» bzw. «gut» in die Haut einzieht. Die Versorgung der Haut mit Fett wurde am schlechtesten beurteilt - die Qualität bewerteten nur 9 Probanden $(31,0 \%)$ mit «sehr gut» und 14 Probanden mit «gut» $(48,3 \%)$, während 4 Probanden $(6,9 \%)$ die Fettversorgung mit «ausreichend» und 2 mit «schlecht» bewerteten.

In Bezug auf unerwünschte Wirkungen gaben 22 Probanden $(75,9 \%)$ an, keine Nebeneffekte beobachtet zu haben, während 6 Probanden $(20,7 \%)$ während der Benutzung der Creme unerwünschte Wirkungen in Form von vorübergehenden allergischen Reaktionen, Hautirritationen und -rötungen angaben. Ein Proband (3,4\%) machte dazu keine Angaben. Aufgrund fehlender Mindestangaben zu Hautstatus und vorliegenden Erkrankungen war eine Kausalitätsbewertung nicht möglich, sodass die vorliegenden Fälle als «nicht einstufbar» betrachtet werden müssen.

\section{Prospektive Vorher-nachher-Befragung}

Für Veränderungen im Hautzustand mit den Items «weich-hart» und «trocken-feucht» gab es signifikante Ergebnisse (t-Test: $p=0,04$ für «weich-hart» und $p=0,01$ für «trocken-feucht»). Alle anderen Items zeigten keine signifikanten Unterschiede (Abb. 6). Die Post-hoc-Poweranalyse ergab unter einem Signifikanzniveau von 5\% für die Veränderung der Hautfeuchtigkeit («trocken-feucht») eine Power von $95 \%$.

Abbildung 7 zeigt die Veränderungen bei den Hautproblemen. Obwohl in der Grafik eine Verbesserung in den Items «Jucken», «empfindlich», und «schuppt» deutlich zu erkennen ist, reicht dennoch die geringe Fallzahl (6 Probandinnen) nicht aus, um eine statistische Signifikanz zu errechnen. So konnten für keine der Items signifikante Änderungen festgestellt werden. Lediglich beim Item «rissig» ist ein statistischer Trend zu sehen (t-Test: $\mathrm{p}=0,08)$. 


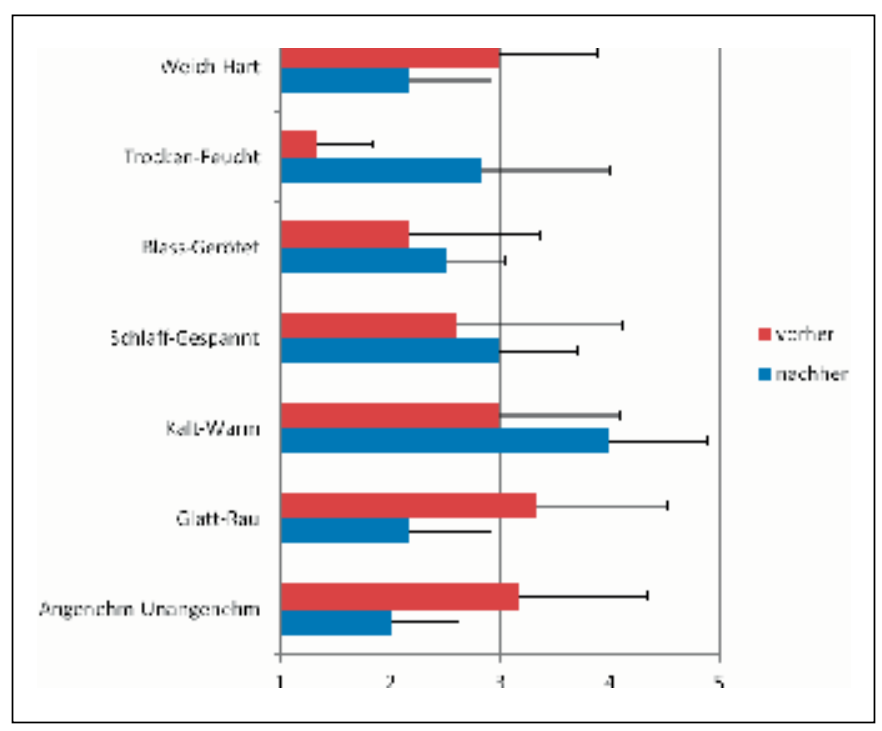

Abb. 6. Veränderungen im Hautzustand der Befragten auf einer Skala von 1 bis 5, wobei ein Wert von 3 die Mitte der Gegensatzpaare markiert. Darstellung als Mittelwert mit Standardabweichung.

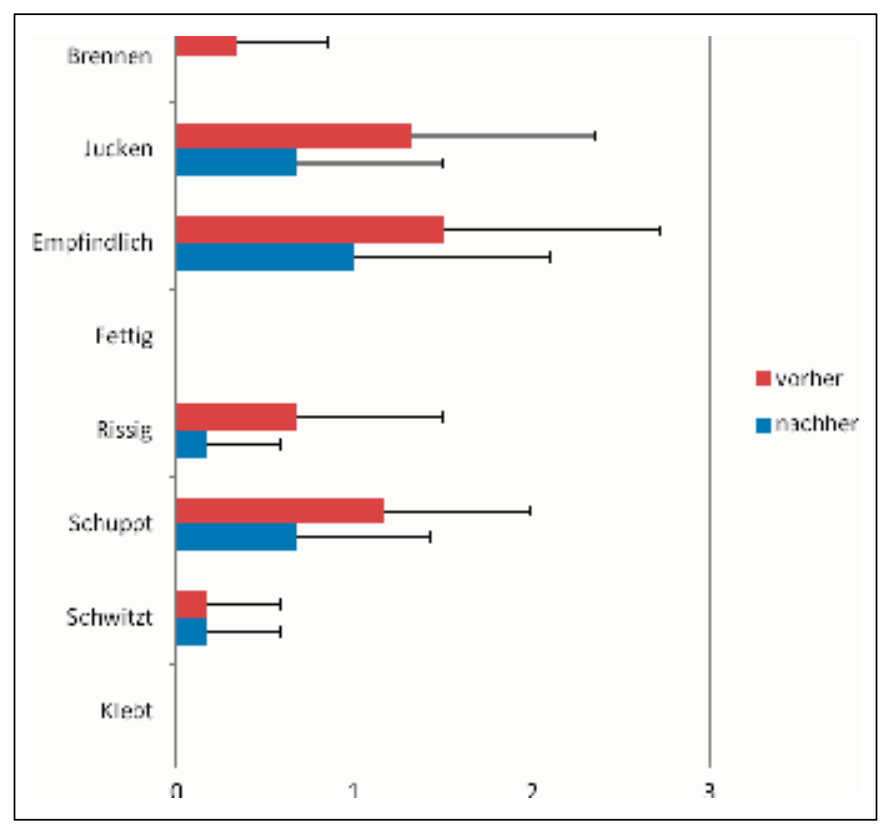

Abb. 7. Veränderung bei den Hautproblemen der Befragten auf einer Skala von $3=$ «sehr stark», 2 = «stark», $1=$ =mäßig», $0=$ «gar nicht», «nicht relevant» bzw. «Frage betrifft mich nicht». Darstellung als Mittelwert mit Standardabweichung.

\section{Hautfeuchtigkeitsveränderung mittels Corneometermessung}

Die Studie zur Hautfeuchtigkeitsverbesserung mittels Corneometertestung fand an 6 gesunden freiwilligen Probandinnen (mittleres Alter 49,0 $\pm 11,0$ Jahre) mit trockener $(\mathrm{n}=5)$ oder sehr trockener Haut $(\mathrm{n}=1)$ statt. Im Prä-post-Vergleich zeigte die Mesem-Creme in der Veränderung der Hautfeuchtigkeit signifikante Effekte ( $\mathrm{p}=0,011, \mathrm{t}$-Test; $25,8 \pm 5,8$ vorher zu 46,6 \pm 9,2 nachher) im Vergleich zur unbehandelten Haut

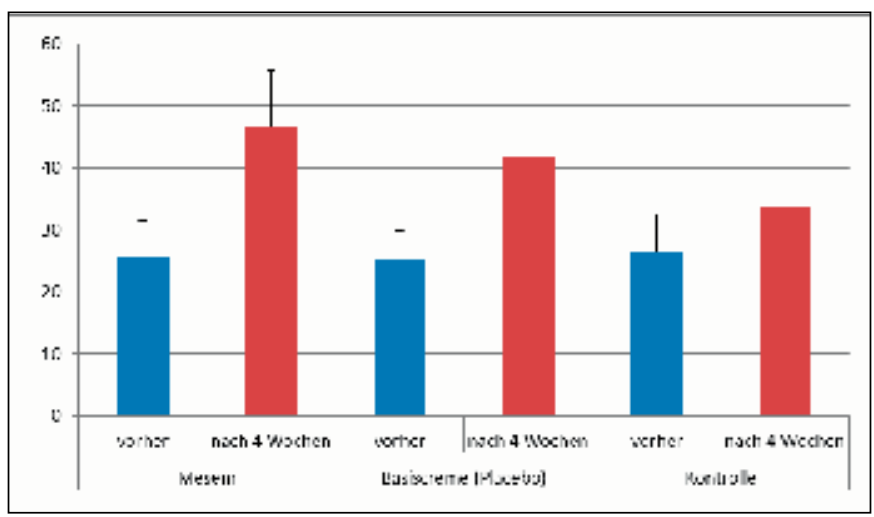

Abb. 8. Verbesserung der Hautfeuchtigkeit im Corneometertest gemessen in arbiträren Einheiten (AU). Darstellung als Mittelwert mit Standardabweichung.

$(26,3 \pm 6,0$ vorher zu 33,8 $\pm 6,0$ nachher $)$, sowie einen statistischen Trend ( $\mathrm{p}=0,11$, $\mathrm{t}$-Test) im Vergleich mit der Basiscreme ohne Mesem-Zusatz $(25,1 \pm 4,7$ vorher zu 41,9 \pm 7,3 nachher). Insgesamt wäre eine Fallzahl von $n=47$ Probanden notwendig gewesen, um die Differenz zwischen Basiscreme und Mesem-Creme signifikant abzusichern.

Prozentual führte die Mesem-Creme zu einer Anreicherung der Hautfeuchtigkeit nach 4-wöchiger Anwendung um durchschnittlich 80,8\%. Die wirkstofffreie Grundlage (Placebo) führte im gleichen Untersuchungszeitraum $\mathrm{zu}$ einer Feuchtigkeitsanreicherung von 66,8 \%, während in der unbehandelten Kontrolle eine Witterungsbedingt leichte Verbesserung der Hautfeuchtigkeit von 28,3\% gemessen wurde. Abbildung 8 zeigt diesen Zusammenhang noch einmal grafisch auf. Während der Testung wurden keine Unverträglichkeiten in Form von Hautreizungen oder -rötungen bei den Probanden beobachtet.

\section{Schlussfolgerung}

In dieser empirischen Arbeit wurde erstmals die MesemCreme, eine Creme mit einem Pflanzenextrakt aus M. crystallinum, umfassend durch eine Corneometermessung und 2 Anwendungsbeobachtungen im Hinblick auf Wirkung, Produkteigenschaften und Verträglichkeit evaluiert. Ein zentraler Aspekt war dabei die Hautfeuchtigkeitsanalyse mittels Corneometermessung. Hier konnte nur im Vergleich zur unbehandelten Haut eine signifikante Steigerung der Hautfeuchtigkeit erzielt werden. Im Vergleich mit der Basiscreme lag lediglich ein statistischer Trend vor. Die Steigerung der Hautfeuchtigkeit wurde auch in den Anwendungsbeobachtungen verifiziert. In der retrospektiven Nutzerbefragung wurde der Creme ein deutliches Wirkungsprofil in Bezug auf die Hautfeuchtigkeit von den Nutzern bescheinigt. Auch die Anwendungsbeobachtung bei einer Kohorte von 6 Rollstuhlfahrern ergab einen deutlichen Effekt in die gleiche Richtung. Ein 
Vergleich mit der Literatur ergab, dass eine bereits auf dem Markt verfügbare Mittagsblumen-Creme über ein ähnliches Wirkungsprofil verfügt [15]. Diese Mittagsblumen-Creme wurde im Rahmen einer Anwendungsbeobachtung bei 23 Probanden mit trockener bis sehr trockener Haut in einem ähnlichen Versuchsaufbau mit Corneometertestung untersucht. Dabei wurde die Hautfeuchtigkeit innerhalb der ersten $8 \mathrm{~h}$ alle $2 \mathrm{~h}$ nach Applikation sowie nach 7 Tagen gemessen. Im Gegensatz zu der vorliegenden Studie kamen dabei eine Mittagsblumen-Lotion und eine Creme zum Einsatz, während auf ein wirkstofffreies Präparat verzichtet wurde. Als Kontrolle kam, wie in der vorliegenden Studie, eine unbehandelte Hautstelle zum Einsatz. Auch hier führte die Anwendung der Mittagsblumen-Creme zu einer signifikanten Steigerung der Hautfeuchtigkeit im Vergleich zur Kontrolle von ca. 65\%, was mit der in dieser Arbeit gemessenen Differenz von 52,5\% gut übereinstimmt. Allerdings ist dieser Wert nur bedingt vergleichbar, da es sich in diesem Fall um einen direkten Effekt nach $2 \mathrm{~h}$ handelt, während der in der vorliegenden Arbeit durchgeführte Corneometertest die Hautfeuchtigkeit nach 4 Wochen Anwendungszeit untersuchte. Zudem konnte über die Vergleichbarkeit der Rezepturen der angewandten Mittagsblumen-Cremes keine Aussage getroffen werden, da entsprechende Angaben fehlten [15].

Die Verbesserungen der Hautfeuchtigkeit, die in den beiden Anwendungsbeobachtungen beschrieben wurden, sind möglicherweise auch auf die Wirkung der Basiscreme zurückzuführen. Der Einfluss des Mittagsblumenextrakts kann vor dem Hintergrund der Ergebnisse der Corneometermessung derzeit nicht abschließend beurteilt werden. Unter Berücksichtigung der Ergebnisse von Ibtissem et al. [5] und Greco [15] kann allerdings von einer Wirkung auf die Hautfeuchtigkeit ausgegangen werden. Um diesen Effekt statistisch belastbar nachzuweisen, sind größere Studien notwendig. Diese sollten sich spezifisch auf die in den vorliegenden Studien relevanten Parameter der Hautfeuchtigkeit konzentrieren.

In der retrospektiven Befragung ist vor allem die geringe Rücklaufquote kritisch zu bewerten. Gegebenenfalls sind da- durch Erfahrungswerte mit der Anwendung der MesemCreme nicht mitgeteilt worden. Es handelt sich sowohl in der Altersstruktur als auch in der Anwendung der Mesem-Creme um ein sehr heterogenes Profil von Anwendern, das Menschen aller Altersgruppen vom Kleinkind bis zum Senioren einschloss. Auch das Hautprofil der Studienteilnehmer war uneinheitlich. Neben Hauterkrankungen wie Neurodermitis oder Psoriasis wurden von den Teilnehmern auch leichte Hautirritationen («trockene Haut») berichtet, was zum Teil in einem hohen Anteil neutraler Antworten resultierte («Frage betrifft mich nicht»). In beiden Anwendungsbeobachtungen wären andere Fragebogeninstrumente besser geeignet gewesen, um das Wirkungsprofil der Mesem-Creme präziser abzubilden.

Möglicherweise können künftige Studien mit klarerem Zuschnitt und größeren Fallzahlen durchgeführt werden, um den Effekt der Mesem-Creme besser zu fokussieren, in dem der Hautstatus der Teilnehmer im Vorfeld enger eingegrenzt wird. So zeigte eine aktuelle epidemiologische Studie, dass Hauterkrankungen bei Bauarbeitern gehäuft auftreten [17]. Allerdings konnte nach Sichtung der einschlägigen Literatur kein Hinweis auf entsprechende Studien z.B. zur Wirkung von Handcremes bei Bauarbeitern gefunden werden. Hier wäre es sinnvoll, eine Studie an einer Teilnehmergruppe mit eng definierten Hautproblemen durchzuführen.

In Bezug auf die Grundlagenforschung sind ebenfalls weitere Studien nötig. So wurde der hier verwandte reine Presssaft bisher nicht in Bezug auf seine Eigenschaften untersucht. Dies könnte in weiteren Studien oder anderen experimentellen Modellen nachgeholt werden, um die Aussagen zur Hautverträglichkeit und Wirkung der Mesem-Creme weiter abzusichern.

\section{Disclosure Statement}

Die Autoren erklären hiermit, dass keine Interessenskonflikte in Bezug auf das vorliegende Manuskript bestehen.

\section{Literatur}

1 Scharte J: Charakterisierung von zwei Stressinduzierbaren Serin-Threonin-Proteinkinasen und eines Transkriptionsfaktors der AP2-EREBPFamilie bei Mesembryanthemum crystallinum L. Dissertation, Universität Münster, 2002.

2 Bernard S: Die Pflanzenwelt Lanzarotes (Teil 6) Die Kristall-Mittagsblume - Wundermittel für die Haut. Lanzarote $37^{\circ}$ 2009;35:30-33.

3 Madaus G: Lehrbuch der Biologischen Heilmittel. Leipzig, Thieme, 1938.

4 Wende K, Stolle R, Lindequist U, Grünewald A, Meyer U: Dank Trockenstress zum Hautpflegemittel. Pharm Ztg 2006;151:322-324.

5 Ibtissem B, Imen M, Souad S: Dosage of 2,6-Bis (1.1-Dimethylethyl)-4-Methylphenol (BHT) in the Plant Extract Mesembryanthemum crystallinum. J Biomed Biotechnol 2010;2010:142486.
6 Cushman JC, Bohnert HJ: Induction of Crassulacean acid metabolism by salinity - molecular aspects; in Läuchli A, Lüttge U (eds): Salinity: Environment - Plants - Molecules. Rotterdam, Springer, 2000, pp 361-393.

7 Brinckmann E, Wartinger M, von Willert D: Turgoränderungen in Blasenzellen von Mesembryanthemaceen. Berichte Dtsch Bot Ges 1985;98:447-454.

8 Cosentino $\mathrm{C}: \mathrm{Na}+\mathrm{H}+$ transporters of the halophyte Mesembryanthemum crystallinum L. Dissertation, Technische Universität Darmstadt, 2008.

9 Lieb JWF: Die Eispflanze als ein fast specifisches Arzneymittel empfohlen. Im Verlag der Vierlingischen Buchhandlung, 1785. https://opacplus.bsbmuenchen.de/metaopac/search? View $=$ default $\& d b=$ 100\&id=BV00144564810 (Zugriff 19.11.14).
10 Hahnemann S: Apothekerlexikon: L- P. Crusius, 1798. http://books. google.de/books?id=sUI9AAAAcAAJ $\&$ printsec $=$ frontcover $\& h l=d e \&$ source $=g b s \_g e \_s u m m a r y$ $r \&$ cad $=0 \# v=$ onepage $\& q \& f=$ falsehttp://books. google.de/books?id $=j Y R j R A A A C A A J$ (Zugriff 19.11.14).

11 Lincke CG: Vollständiges Recepttaschenbuch: in alphabetischer Ordnung. Gebhardt und Reisland, 1841. http://books.google.de/books?id=9RI9AAAAcAAJ $\& p g=P A 460 \& l p g=P A 460 \& d q=$ Lincke $+C G:+$ Vollst $\%$ C3\%A4ndiges + Recepttaschenbuch, \&source $=$ bl\&ots= D_8wWIAPUE\&sig=Bs8uq6-4cwMROziCX_YX_ $f 1 w V s I \& h l=d e \& s a=X \& e i=8 J R s V P f l O M r e P b G F g a g K$ \&ved $=0 C C U Q 6 A E w A A \# v=$ onepage $\& q=$ Lincke $\% 20 C G \% 3 A \% 20$ Vollst $\%$ C3 $\%$ A 4ndiges $\% 20$ Recepttaschenbuch\%2C\&f=false (Zugriff 19.11.14). 
12 Most GF: Enzyklopädie der gesammten Volksmedizin. Brockhaus; 1843. www.textlog.de/volksmedizin. html (Zugriff 19.11.14).

13 Raak C: Eigenschaften, Verträglichkeit und Wirkung der Mesem-Creme (Mesembryanthemum crystallinum L.) bei Hautproblemen unterschiedlicher Genese. Berlin, Hochschule für Gesundheit und Sport, Technik und Kunst, 2014.
14 Bouftira I, Abdelly C, Sfar S: Characterization of cosmetic cream with Mesembryanthemum crystallinum plant extract: influence of formulation composition on physical stability and anti-oxidant activity. Int J Cosmet Sci 2008;30:44-52.

15 Greco A: Kristall-Mittagsblume: Trockenpflanze zur medizinischen Hautpflege. Pharm Ztg 2010; 155:57-59.
6 Heinrich U, Koop U, Leneveu-Duchemin M, et al.: Multicentre comparison of skin hydration in terms of physical- physiological- and product-dependent parameters by the capacitive method (Corneometer CM 825). Int J Cosmet Sci 2003;25:45-53.

17 Timmerman JG, Heederik D, Spee T, Smit LAM: Skin symptoms in the construction industry: occurrence and determinants: skin symptoms in the construction industry. Am J Ind Med 2014;57:660-668.

\section{0 prägnante Pflanzenporträts}

mit großem Praxisnutzen

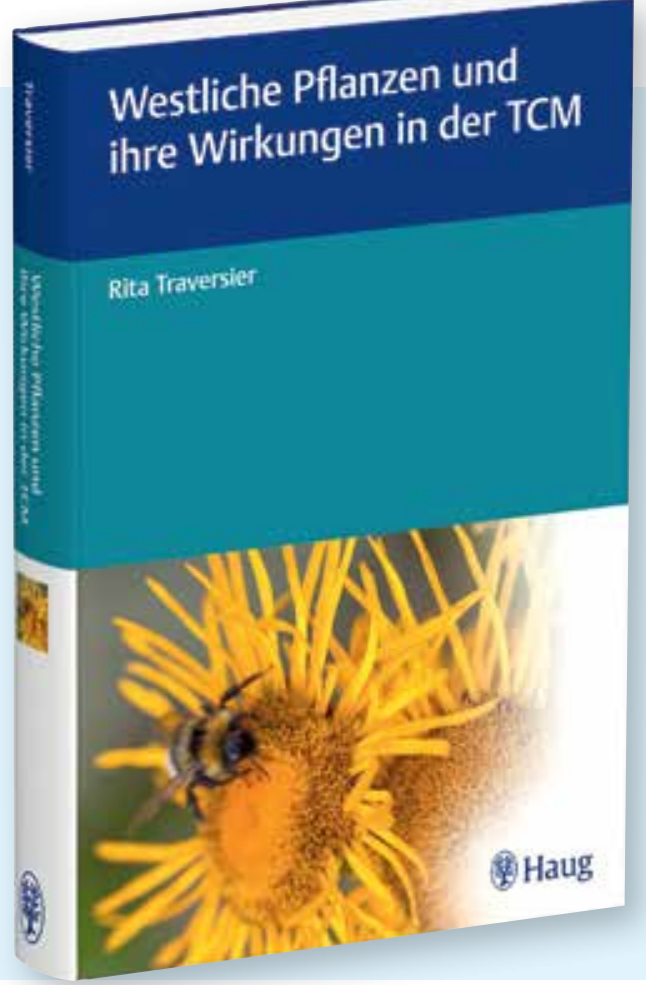

\section{Lesen, Sehen, Umsetzen}

Die 140 Pflanzenporträts bringen die relevanten Eigenschaften, die therapeutische Wirkung und die Anwendungsbereiche sowie mögliche Darreichungsformen der wichtigsten westlichen Heilpflanzen aus Sicht der TCM auf den Punkt.

Jede Pflanze ist mit Foto und Grafik illustriert so sehen Sie auf einen Blick, in welchem Organsystem die jeweilige Pflanze wirksam ist.

Ein praktisches Handbuch, das Ihnen den täglichen Einsatz in der Praxis erleichtert!

R. Traversier Westliche Pflanzen und ihre Wirkungen in der TCM 2014, ca. 464 S., ca. 280 Abb., geb. ISBN 978-3-8304-7662-7 $69,99 €[D]$ $72,00 €[A], 98,00 \mathrm{CHF}$

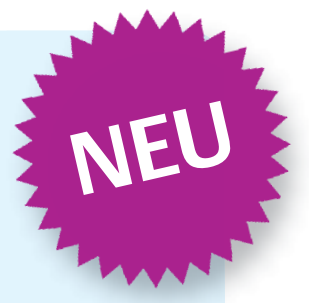

\section{Versandkostenfreie Lieferung innerhalb Deutschlands!}

